\title{
Noise Induced Propagation in Monostable Media
}

\author{
A. A. Zaikin, ${ }^{1}$ J. García-Ojalvo, ${ }^{2}$ L. Schimansky-Geier, ${ }^{3}$ and J. Kurths ${ }^{1}$ \\ ${ }^{1}$ Institut für Physik, Potsdam Universität, Am Neuen Palais 10, D-14469 Potsdam, Germany \\ ${ }^{2}$ Departament de Física i Enginyeria Nuclear, Universitat Politècnica de Catalunya, Colom 11 E-08222 Terrassa, Spain \\ ${ }^{3}$ Institut für Physik, Humboldt Universität zu Berlin, Invalidenstrasse 110, D-10115 Berlin, Germany
}

(Received 24 April 2001; published 19 December 2001)

\begin{abstract}
We show that external fluctuations are able to induce propagation of harmonic signals through monostable media. This property is based on the phenomenon of doubly stochastic resonance, where the joint action of multiplicative noise and spatial coupling induces bistability in an otherwise monostable extended medium, and additive noise resonantly enhances the response of the system to a harmonic forcing. Under these conditions, propagation of the harmonic signal through the unforced medium is observed for optimal intensities of the two noises. This noise-induced propagation is studied and quantified in a simple model of coupled nonlinear electronic circuits.
\end{abstract}

DOI: $10.1103 /$ PhysRevLett.88.010601

It is a well-established fact nowadays that dynamical noise, which usually has a disordering impact, can be used to induce order in nonlinear nonequilibrium systems under certain conditions. Examples of this counterintuitive influence of random fluctuations are noise-induced transitions [1-4], stochastic transport in ratchets [5] (also in a synthesis with a transition [6]), or noise-induced pattern formation [7]. However, one of the most far-reaching examples is stochastic resonance (SR) [8], which has been experimentally observed in several physical and biological systems [9]. In the classical situation, SR consists of an optimization by noise of the response of a bistable system to a weak periodic signal. Besides this standard scenario, SR has also been found in monostable [10], excitable [11], nondynamical [12], and thresholdless [13] systems, in systems without an external force (what is called coherence resonance) $[14,15]$, and in systems with transient noiseinduced structure [16].

Additionally, it has been recently shown that the energy of fluctuations can be used even more efficiently in spatially extended systems, by using noise twofold: to synchronize output hops across a potential barrier with an external signal, and also to optimally construct the barrier itself. This phenomenon is known as doubly stochastic resonance (DSR) [17]. DSR occurs in systems of coupled overdamped oscillators; and it is a synthesis of two basic phenomena: SR and noise-induced phase transitions [18]. Another important and nontrivial phenomenon connected with SR in spatially distributed systems is the phenomenon of noise enhanced propagation, in which the propagation of a harmonic forcing through an unforced bistable or excitable medium is increased for an optimal intensity of the additive noise $[19,20]$.

In this Letter, we present a new propagation phenomenon in monostable media. We show that noise can enhance propagation in deterministically monostable media, without any deterministic threshold, provided bistability is induced by a second (multiplicative) noise and coupling through a phase transition. Although numerous works
PACS numbers: 05.40.Ca, 05.70.Fh

about noise-induced propagation exist (e.g., [21]), to our knowledge propagation in monostable media, which is a very important class of dynamical systems, has not been considered before. In what follows, we present this propagation or in a general model of overdamped coupled nonlinear oscillators. Subsequently, and for the sake of concreteness, the phenomenon is analyzed in particular in a simple model of coupled electronic circuits.

We study a general class of spatially distributed systems, which are locally coupled and periodically forced:

$$
\begin{aligned}
\dot{x}_{i}= & f\left(x_{i}\right)+g\left(x_{i}\right) \xi_{i}(t)+\frac{D}{4} \sum_{j \in n n(i)}\left(x_{j}-x_{i}\right) \\
& +\zeta_{i}(t)+A_{i} \cos (\omega t+\varphi),
\end{aligned}
$$

where $x_{i}$ is defined in a two-dimensional discrete space of $N \times N$ cells, with $i$ denoting the cell position $\left[i=i_{x}+N\left(i_{y}-1\right)\right.$, where $i_{x}$ and $i_{y}$ run from 1 to $N]$. The sum in Eq. (1) runs over all nearest neighbors of site $i[n n(i)]$. The additive and multiplicative noise terms are mutually uncorrelated Gaussian distributed with zero mean, and white both in space and time, i.e., $\left\langle\zeta_{i}(t) \zeta_{j}\left(t^{\prime}\right)\right\rangle=\sigma_{a}^{2} \delta_{i j} \delta\left(t-t^{\prime}\right)$ and $\left\langle\xi_{i}(t) \xi_{j}\left(t^{\prime}\right)\right\rangle=$ $\sigma_{m}^{2} \delta_{i j} \delta\left(t-t^{\prime}\right)$. The results are averaged over the initial phase $\varphi$ of a harmonic forcing, which has amplitude $A_{i}$ and frequency $\omega$.

In the absence of periodic forcing $\left(A_{i}=0\right)$, different types of noise-induced phase transitions can be obtained for different forces $f\left(x_{i}\right)$ and $g\left(x_{i}\right)$ [3]. In particular, a system with a monostable deterministic potential can undergo a phase transition to a noise-induced bistable state for a suitable stochastic forcing $g\left(x_{i}\right)$ [18]. There, in the presence of a global harmonic forcing, DSR is observed [17]. We consider in this Letter the case that the periodic forcing is applied coherently along only one side, as shown in Fig. $1\left[A_{i}=A\left(\delta_{i_{x}, 1}+\delta_{i_{x}, 2}+\delta_{i_{x}, 3}\right)\right]$, and study the propagation of this forcing action into the nonexcited portion of the system.

Even though the results shown below are very general, for a quantitative study we choose particular functions 


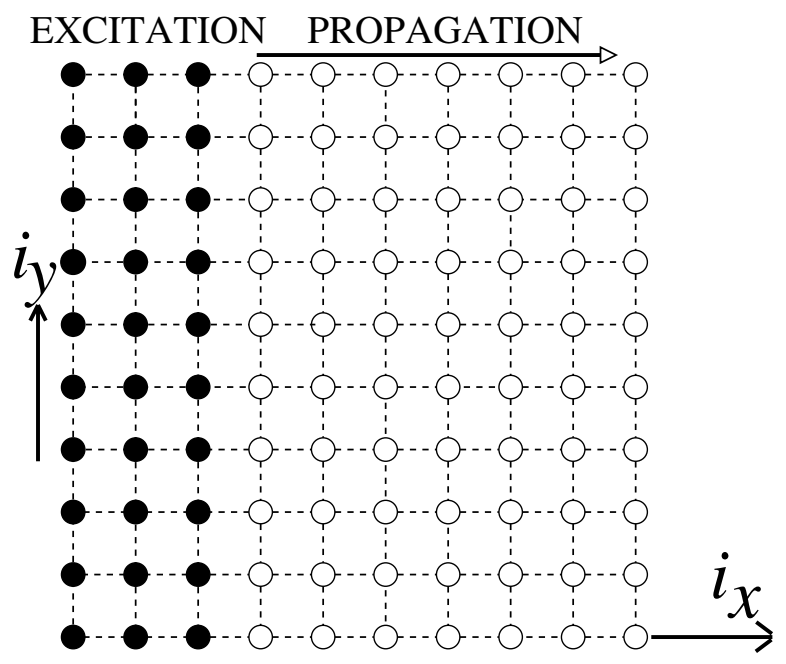

FIG. 1. Scheme of the spatially distributed system. The periodic excitation is performed only from one side, elements under the direct periodic action are denoted by black. All oscillators are under the influence of noise. To study the behavior of both driven and nondriven elements, first three columns $\left(i_{x}=1,2,3\right)$ are periodically driven; however, to achieve propagation it is sufficient to excite only one column.

$f(x)$ and $g(x)$. These functions model the local dynamics of the electronic circuit designed theoretically (i.e., it is so far a thought experiment) and displayed in Fig. 2. This circuit consists of a capacitor with capacitance $C$, a time-varying resistor (TVR) with conductance $G(t)$, a current generator $I(t)$, four coupling resistors $R_{c}$ (responsible for the diffusive coupling with the neighbors), and a nonlinear resistor $R_{N}$, which is realized with a set of ordinary diodes or operational amplifiers [22], and has the characteristic function

$i_{N}=h(V)= \begin{cases}G_{b} V+\left(G_{a}-G_{b}\right) B_{p}, & \text { if } V \leq-B_{p}, \\ G_{a} V, & \text { if }|V|<B_{p}, \\ G_{b} V-\left(G_{a}-G_{b}\right) B_{p}, & \text { if } V \geq B_{p},\end{cases}$

where $i_{N}$ is the current through the nonlinear resistor $\left(R_{N}\right)$, $V$ is the voltage drop across it, and the parameters $G_{a}, G_{b}$, and $B_{p}$ determine the slopes and the break point of its piecewise-linear characteristic curve.

We now consider that the conductance of the TVR fluctuates randomly in time $\left[G_{i}(t)=\xi_{i}(t)\right]$, and that the input

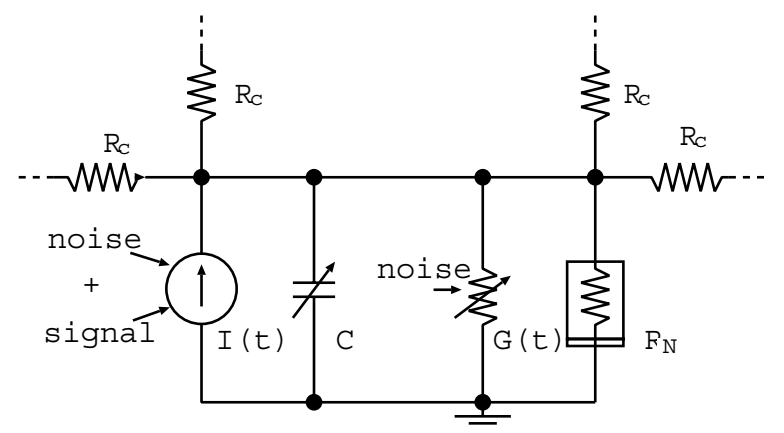

FIG. 2. Nonlinear electronic circuit at element $i$. current $I(t)$ has the form of a periodic signal to which an uncorrelated Gaussian noise $\zeta(t)$ is added $\left[I_{i}(t)=\zeta_{i}(t)+\right.$ $\left.A_{i} \cos (\omega t+\varphi)\right]$. Under these conditions, the dynamics of the spatially coupled system is described by Eq. (1), where $x_{i}$ now represents the voltage drop across the nonlinear resistor of circuit $i$, and the forces are $f(x)=-h(x)$ and $g(x)=x$ [22]. Additionally, $C=1$ by an appropriate time normalization, and the coupling strength $D=\frac{4}{C R_{c}}$.

SR behavior can be expected if the system is bistable for the chosen set of parameters. Regions of bistability can be determined approximately by means of a standard mean-field procedure [3]. The mean-field approximation consists of replacing the nearest-neighbor interaction by a global term in the Fokker-Planck equation corresponding to (1) in the absence of external forcing. In this way, we get the steady-state probability distribution $P_{\mathrm{st}}$ :

$$
\begin{aligned}
P_{\text {st }}(x, m)= & \frac{C(m)}{\sqrt{\sigma_{m}^{2} g^{2}(x)+\sigma_{a}^{2}}} \\
& \times \exp \left(2 \int_{0}^{x} \frac{f(y)-D(y-m)}{\sigma_{m}^{2} g^{2}(y)+\sigma_{a}^{2}} d y\right),
\end{aligned}
$$

where $C(m)$ is a normalization constant and $m$ is the mean field, defined implicitly by:

$$
m=\int_{-\infty}^{\infty} x P_{\mathrm{st}}(x, m) d x .
$$

The value of $m$ is obtained by the self-consistent solution of Eq. (4), which enables us to determine the transition lines between the ordered bistable $(m \neq 0)$ and the disordered monostable $(m=0)$ phases. These transition boundaries are shown in Fig. 3 in the $\left(D, \sigma_{m}^{2}\right)$ plane for three different values of the additive noise intensity. Note that bistability requires both multiplicative noise and coupling between elements. We also find that an increase in additive noise reduces the bistable region. This gives DSR a special character with respect to standard SR [17].

Now, we place ourselves within the bistable regime supported by multiplicative noise and coupling (e.g.,

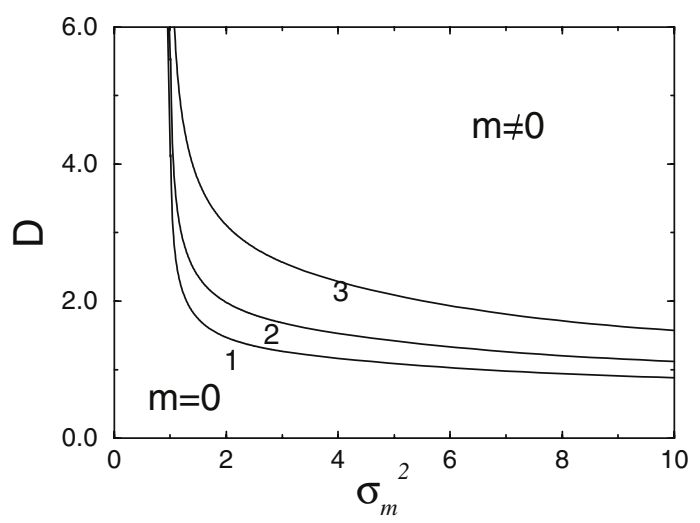

FIG. 3. Mean-field transition lines between disordered monostable $(m=0)$ and ordered bistable $(m \neq 0)$ phases for model (1): $\sigma_{a}^{2}=0.3$ (label 1), $\sigma_{a}^{2}=0.5$ (label 2), and $\sigma_{a}^{2}=1.0$ (label 3). Here $G_{a}=0.5, G_{b}=10$, and $B_{p}=1$. 
$D=3, \sigma_{m}^{2}=3$ ), and investigate the propagation of a wave through the system. To that end, we harmonically excite the lattice from one side, as shown in Fig. 1, with boundary conditions periodic in the vertical direction and no-flux in the horizontal direction. The propagation will be quantified by the system's response at the excitation frequency, computed as $Q^{(j)}=\sqrt{\left[Q_{\mathrm{sin}}^{(j)}\right]^{2}+\left[Q_{\mathrm{cos}}^{(j)}\right]^{2}}$, with

$$
\begin{aligned}
& Q_{\text {sin }}^{(j)}=\frac{\omega}{n \pi} \int_{0}^{2 \pi n / \omega} 2 m_{j}(t) \sin (\omega t) d t, \\
& Q_{\mathrm{cos}}^{(j)}=\frac{\omega}{n \pi} \int_{0}^{2 \pi n / \omega} 2 m_{j}(t) \cos (\omega t) d t,
\end{aligned}
$$

where $m_{j}(t)$ is the field (voltage) averaged along the vertical column (Fig. 1), i.e., $m_{j}(t)=\frac{1}{N} \sum_{k=1}^{N} x_{j+(k-1) N}(t)$.

The value of $Q^{(j)}$ for different oscillators along the chain is shown in Fig. 4(a), for increasing intensities of additive noise within the noise-induced bistable regime. The forcing amplitude is taken to be large enough to produce hops between the two wells in the bistable oscillators, without the need of additive noise. Therefore, for the first oscillators an increase of additive noise leads only to a decreasing response at the forcing frequency, whereas for distant oscillators the situation changes qualitatively. There, a response is induced that depends nonmonotonically on the additive noise intensity. Clearly, a certain amount of additive noise exists for which propagation of the harmonic signal is optimal. For smaller $\sigma_{m}^{2}$ [Fig. 4(b] the system leaves the bistable region; hence the response is small and always monotonically decreasing. Hence, the resonantlike
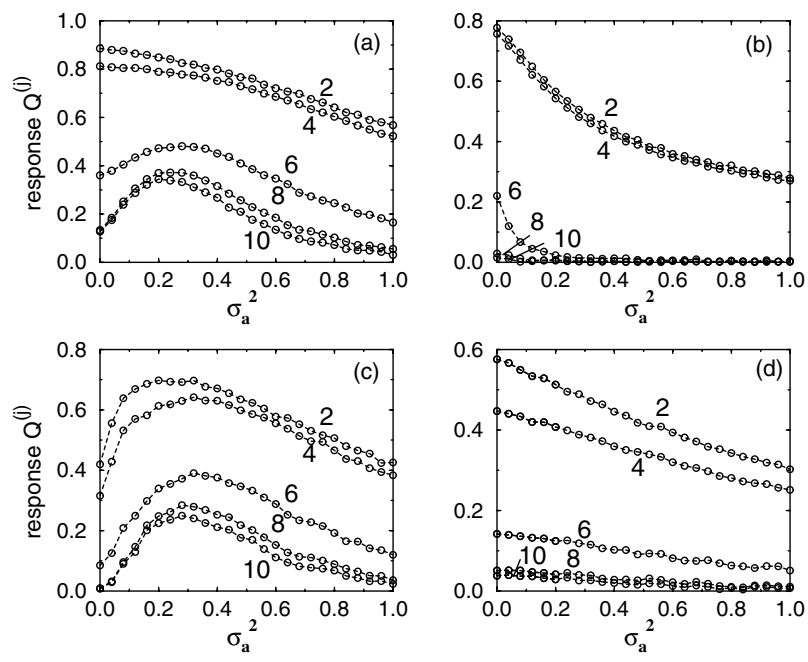

FIG. 4. Response $Q^{(j)}$ to a periodic excitation in different columns (the order $j$ is shown in the curve labels) vs additivenoise intensity (a), (c) inside the bistability region $\left(\sigma_{m}^{2}=3\right)$, and (b),(d) outside that region $\left(\sigma_{m}^{2}=0.5\right)$. As shown in Fig. 1, the oscillators with index $j=i_{x}=1,2,3$ are directly excited by the periodic force, and oscillators with $j=i_{x}>3$ are excited through the excitation propagation. Parameters are those of Fig. 3, and $D=3$. The amplitude is: (a),(b) $A=0.3$ (noiseinduced propagation) and (c),(d) $A=0.2$ (spatiotemporal doubly stochastic resonance). effect requires suitable intensities of both the additive and multiplicative noises.

A propagation of the harmonic signal can also be obtained for values of the forcing amplitude small enough so that hops are not produced in the directly excited sites in the absence of additive noise. This is the regime in which DSR really occurs in the excited part of the system, and the excitation propagates through the rest of the lattice enhanced by noise. Now all the oscillators have a nonmonotonic dependence on the additive noise intensity for a multiplicative noise within the bistable region [Fig. 4(c)], and a monotonic one for a multiplicative noise within the monostable region [Fig. 4(d)]. The former case corresponds to a spatiotemporal propagation in the DSR medium, and we call this phenomenon spatiotemporal doubly stochastic resonance (SDSR).

The mechanism of this phenomenon can be explained theoretically on the basis of a mean-field approximation. We give a first qualitative glimpse of this analysis in what follows; quantitative details will be published elsewhere. Because of coupling and multiplicative noise, the system becomes bistable with the behavior approximately governed by a mean-field effective potential [17]

$$
U_{\text {eff }}(x)=U_{0}(x)+U_{\text {noise }}=-\int f(x) d x-\frac{\sigma_{m}^{2} x^{2}}{4} .
$$

Now the effect can be understood in the frame of a standard SR mechanism [8], where the external signal is provided by the periodic force for the directly excited oscillators, and by the influence of the left neighbors for the nonexcited oscillators. For large forcing, only the latter need an additive noise to hop synchronously between wells, whereas for small forcing, both the excited and the nonexcited oscillators display SR. These two behaviors correspond to Figs. 4(a) and 4(c), respectively.

At this point it is worth making several remarks to the phenomenon described above. First, SDSR and noiseinduced propagation in monostable media are strongly different to spatiotemporal SR [23] or noise enhanced propagation [19] in bistable media. The effect presented here can be controlled by multiplicative noise, which modifies the depth and separation of the two potential wells. Therefore, an optimal amount of multiplicative noise is required to support the bistable structure. Nothing similar occurs in array-enhanced SR [24] or in SR in extended bistable systems [25]. On the other hand, an increase of additive noise also leads to a loss of bistability (see Fig. 3), and hence a decrease of $Q$ for large additive noise is explained not only by the fact that disordered hops are produced by intense noise, as in standard SR, but also by the loss of bistability. Second, noise-induced propagation in monostable media is very intriguing from the viewpoint of the theory of extended systems with noise and cannot be directly predicted from DSR. The noise-induced bistability, on which DSR is based, is a collective phenomenon, 
which can be observed only for a positive value of coupling enabling all elements to be close to the same position. In contrast to it, here we have shown that a propagation, which implies that different cells are simultaneously in different states, can occur in such a system without destroying the mechanism of bistability.

In conclusion, we have reported the existence of a propagation phenomenon, in which noise induces wave propagation in monostable media. The joint action of multiplicative noise and spatial coupling induces bistability, and additive noise enhances the propagation of harmonic forcing in the stochastically induced bistable medium. Because of its nontrivial propagation mechanism, this effect can be considered as a contribution to the theory of extended systems with noise. We also expect that these theoretical findings will stimulate experimental work. Especially, such kind of a propagation can be of great importance in communications, due to the fact that the energy of noise is used in a very efficient way, both to construct the potential barrier and to provide propagation enhancement in the noise-supported bistable system. We have demonstrated noise-induced propagation in monostable media in a simple realistic model, but in a general framework. Because of the generality of the model we expect that this effect can be also found in several more complicated real extended systems with noise-induced bistability. Probable experimental implementations include arrays of simple electronic circuits as a communication system [22], analog circuits [26], electronic cellular neural networks [27], and are expected to be achieved in several real spatially distributed systems, such as liquid crystals [28], photosensitive chemical reactions [29], RayleighBénard convection [30], or liquid helium [31].

A.Z. acknowledges financial support from CESCACEPBA through the EC IHP Program (HPRI-1999-CT00071), and from ESA (MPA AO-99-030), J. G. O. from DGES (Spain, PB98-0935). J. K. and L. S. G. from SFB 555 (Germany), and J. K. from EC RTN 158.

[1] J. Smythe, F. Moss, and P. McClintock, Phys. Rev. Lett. 51, 1062 (1983).

[2] W. Horsthemke and R. Lefever, Noise-Induced Transitions (Springer, Berlin, 1984).

[3] J. García-Ojalvo and J. M. Sancho, Noise in Spatially Extended Systems (Springer, New York, 1999).

[4] P. Landa, A. Zaikin, V. Ushakov, and J. Kurths, Phys. Rev. E 61, 4809 (2000).

[5] P. Hänggi and R. Bartussek, in Nonlinear Physics of Complex Systems, edited by J. Parisi, S.C. Müller, and W. Zimmermann (Springer, Berlin, 1996).
[6] P. Reimann, R. Kawai, and C. Van den Broeck, P. Hänngi, Europhys. Lett. 45, 545 (1999).

[7] J. García-Ojalvo, A. Hernández-Machado, and J. M. Sancho, Phys. Rev. Lett. 71, 1542 (1993).

[8] R. Benzi, A. Sutera, and A. Vulpiani, J. Phys. A 14, L453 (1981). L. Gammaitoni, P. Hänggi, P. Jung, and F. Marchesoni, Rev. Mod. Phys. 70, 223 (1998).

[9] B. McNamara, K. Wiesenfeld, and R. Roy, Phys. Rev. Lett. 60, 2626 (1988); J. Douglass, L. Wilkens, and L. Pantazelou, Nature (London) 365, 337 (1993).

[10] N. G. Stocks, N. D. Stei, and P. V. E. McClintock, J. Phys. A 26, L385 (1993).

[11] K. Wiesenfeld, D. Pierson, E. Pantazelou, C. Dames, and F. Moss, Phys. Rev. Lett. 72, 2125 (1994).

[12] Z. Gingl, L. Kiss, and F. Moss, Europhys. Lett. 29, 191 (1995).

[13] S. Bezrukov and I. Vodyanoy, in Unsolved Problems of Noise and Fluctuations, edited by D. Abbott and L. Kiss, AIP Conf. Proc. No. 511 (AIP, New York, 2000).

[14] H. Gang, T. Ditzinger, C. Ning, and H. Haken, Phys. Rev. Lett. 71, 807 (1993).

[15] A. Pikovksy and J. Kurths, Phys. Rev. Lett. 78, 775 (1997).

[16] A. Fuliński, Phys. Rev. E 52, 4523 (1995).

[17] A. Zaikin, J. Kurths, and L. Schimansky-Geier, Phys. Rev. Lett. 85, 227 (2000).

[18] C. Van den Broeck, J. M. R. Parrondo, and R. Toral, Phys. Rev. Lett. 73, 3395 (1994).

[19] J. Lindner, S. Chandramouli, A. R. Bulsara, M. Löcher, and W. L. Ditto, Phys. Rev. Lett. 81, 5048 (1998); Y. Zhang, G. Hu, and L. Gammaitoni, Phys. Rev. E 58, 2952 (1998).

[20] S. Kadar, J. Wang, and K. Showalter, Nature (London) 391, 770 (1998); G. Balázsi, L. B. Kiss, and F. E. Moss, in Unsolved Problems of Noise and Fluctuations (Ref. [13]).

[21] J. García-Ojalvo, A. M. Lacasta, and F. Sagués, J. M. Sancho, Europhys. Lett. 50, 427 (2000); R. Perazzo, L. Romanelli, and R. Deza, Phys. Rev. E 61, R3287 (2000); A. C. H. Rowe and P. Etchegoin, Phys. Rev. E 64, 031106 (2001).

[22] A. A. Zaikin, K. Murali, and J. Kurths, Phys. Rev. E 63, 020103(R) (2001).

[23] F. Marchesoni, L. Gammaitoni, and A. R. Bulsara, Phys. Rev. Lett. 76, 2609 (1996); J. M. G. Vilar and J. M. Rubí, Phys. Rev. Lett. 78, 2886 (1997).

[24] J. F. Lindner, B. K. Meadows, W. L. Ditto, M. E. Inchiosa, and A. R. Bulsara, Phys. Rev. Lett. 75, 3 (1995).

[25] H. S. Wio, Phys. Rev. E 54, R3075 (1996); S. Bouzat and H. S. Wio, Phys. Rev. E 59, 5142 (1999).

[26] F. Moss (private communication).

[27] V. Pérez-Muñuzuri, V. Pérez-Villar, and Leon O. Chua, IEEE Trans. 40, 174 (1993); P. Arena, R. Caponetto, L. Fortuna, and A. Rizzo, IEEE Trans. 48, 360 (2001).

[28] S. Kai, T. Kai, and M. Takata, J. Phys. Soc. Jpn. 47, 1379 (1979).

[29] J. Micheau, W. Horsthemke, and R. Lefever, J. Chem. Phys. 81, 2450 (1984).

[30] C. Meyer, G. Ahlers, and D. Cannell, Phys. Rev. A 44, 2514 (1991).

[31] D. Griswold and J. T. Tough, Phys. Rev. A 36, 1360 (1987). 